\title{
An Improved Phantom for Quality Control of Laser Scanner Digitizers in Picture Archival and Communications Systems
}

\author{
Ethan J. Halpern and Peter D. Esser
}

\begin{abstract}
Various quality control $(\mathrm{QC})$ procedures may be used to evaluate image quality for picture archival and communications (PACS) systems. A standard PACS $\mathrm{QC}$ protocol applied on a regular basis is desirable to ensure optimal diagnostic performance. We describe a OC phantom designed especially to test PACS systems that acquire images by digitizing $x$-ray films. The phantom is a sheet of $x$-ray film upon which a digital test pattern is printed. Multiple parameters of image quality are tested, including resolution, contrast, gray scale, geometric distortion, and noise. Individual test patterns are incorporated to detect specific artifacts of laser scanner digitizers. As part of a regular $\mathrm{QC}$ protocol, the phantom provides an objective measurement of change in digital image quality over time, as well as an objective means for comparison with other systems.

Copyright 1991 by W.B. Saunders Company
\end{abstract}

KEY WORDS: quality control, PACS, teleradiology, test phantom, laser scanner, image quality.

Q UALITY CONTROL (QC) of medical imaging systems is essential to ensure a consistent high level of diagnostic accuracy. A broad spectrum of quality assurance protocols are available for the various radiologic imaging modalities. However, there is no widely accepted QC protocol designed specifically for teleradiology and picture archival and communications (PACS) systems. Given the increasingly widespread use of these systems, such a standardized QC protocol would be useful for routine system QC, as well as for objective comparison of different systems.

Several studies ${ }^{1-4}$ have focused on the performance of laser scanner digitizers in digital radiology systems. We have previously described $^{5}$ many interesting artifacts introduced into digital images by laser scanner digitization. Various basic test patterns (eg, line pairs, gray scales, grids, etc) were used in these studies to analyze the respective systems. A pattern developed by the Society of Motion Picture and Television Engineers (SMPTE) ${ }^{6}$ has been used ${ }^{7}$ to test video display monitors for digital radiology systems. The SMPTE pattern has been applied to test film digitizers as well. It provides line pairs to test high-contrast resolution, squares of varying brightness to test gray scale response, and a grid pattern, which is useful to detect geometric distortion. Resolution is tested over a limited range in both the horizontal and vertical axes in all quadrants of the pattern. Gray scale response is tested in the center of the pattern.

Digital image quality depends on a large number of factors. ${ }^{8.11}$ In an earlier work, ${ }^{12}$ we identified several basic elements of image quality that should be examined for all digital radiography systems. These include (1) highcontrast resolution, (2) low-contrast discrimination, (3) linearity of gray scale, (4) geometric distortion, and (5) noise. We described a QC phantom to measure these factors. This phantom expanded on the SMPTE design in several respects. It included high-contrast line pair patterns covering a wider range of resolutions and oriented in both orthogonal and oblique planes, low-contrast patterns with varying background intensities, and multiple gray scale ramps in different regions of the image. The phantom was created by directly exposing a sheet of $\mathrm{x}$-ray film. Various objects (ie, lead strips, step wedges, wire mesh) were interposed between the $\mathrm{x}$-ray source and the film to create the test pattern. The time required to create a single copy of this phantom was approximately 20 minutes.

The goal of the current study was to design an improved QC phantom that could be generated as a digital data file by a computer algorithm. Such a file could then serve as the input for a laser printer, which would create the desired pattern on film. The film phantom would be digitized on a PACS system. The quality of this digitized image would be documented by visual inspection as well as by quantitative analysis of the digital data.

From the Department of Radiology, Center for Computer Applications in Radiology, Columbia Presbyterian Medical Center, New York, NY.

Address reprint requests to Ethan J. Halpern, MD, Department of Radiology, Thomas Jefferson University, $111 \mathrm{~S} 11$ th St, Philadelphia, PA 19107.

Copyright 1991 by Society of Photo-optical Instrumentation Engineers

0897-1889/91/0404-0002\$03.00/0 


\section{PHANTOM DESIGN}

The proposed phantom is shown in Fig 1. A digitized image of this film phantom obtained with a CommView PACS system (AT\&TPhilips, West Long Branch, NJ) is reproduced in Fig 2.

The digital data file for the QC phantom specifies an image 4,984 $\times 4,084$ pixels with 12 bits of data per pixel. This image is printed on a $14 \times 17$-inch film with a $1 / 2$-inch black margin on each side. Thus, the actual size of the test pattern is $13 \times 16$ inches with a maximum density of 6.2 line pairs $(\mathrm{lp}) / \mathrm{mm}$. The right half of the image is the mirror image of the left half with inversion of the gray scale.

High-contrast resolution line pairs surround the periphery of the image, testing resolution in both the horizontal and vertical directions. There are 13 sets of line pairs oriented in the horizontal axis and 14 sets of line pairs oriented in the vertical axis. An additional 15 sets of diagonally oriented line pairs are present just below the upper set of horizontal line pairs. The line pair densities range from approximately $0.5 \mathrm{lp} / \mathrm{mm}$ to $6.2 \mathrm{lp} / \mathrm{mm}$. High-contrast resolution is quantified by noting the finest line pair group that can be clearly distinguished along its entire length on the system being tested. Higher line pair densities are arranged at the periphery of the image to maximally stress the imaging system being tested. The peripheral location of these line pair patterns is also useful to test for barrel and pin cushion distortion.

Low-contrast discrimination is tested with squares of diminishing size $(3.3 \mathrm{~mm}$ down to $0.08 \mathrm{~mm}$ ) embedded in a background of slightly

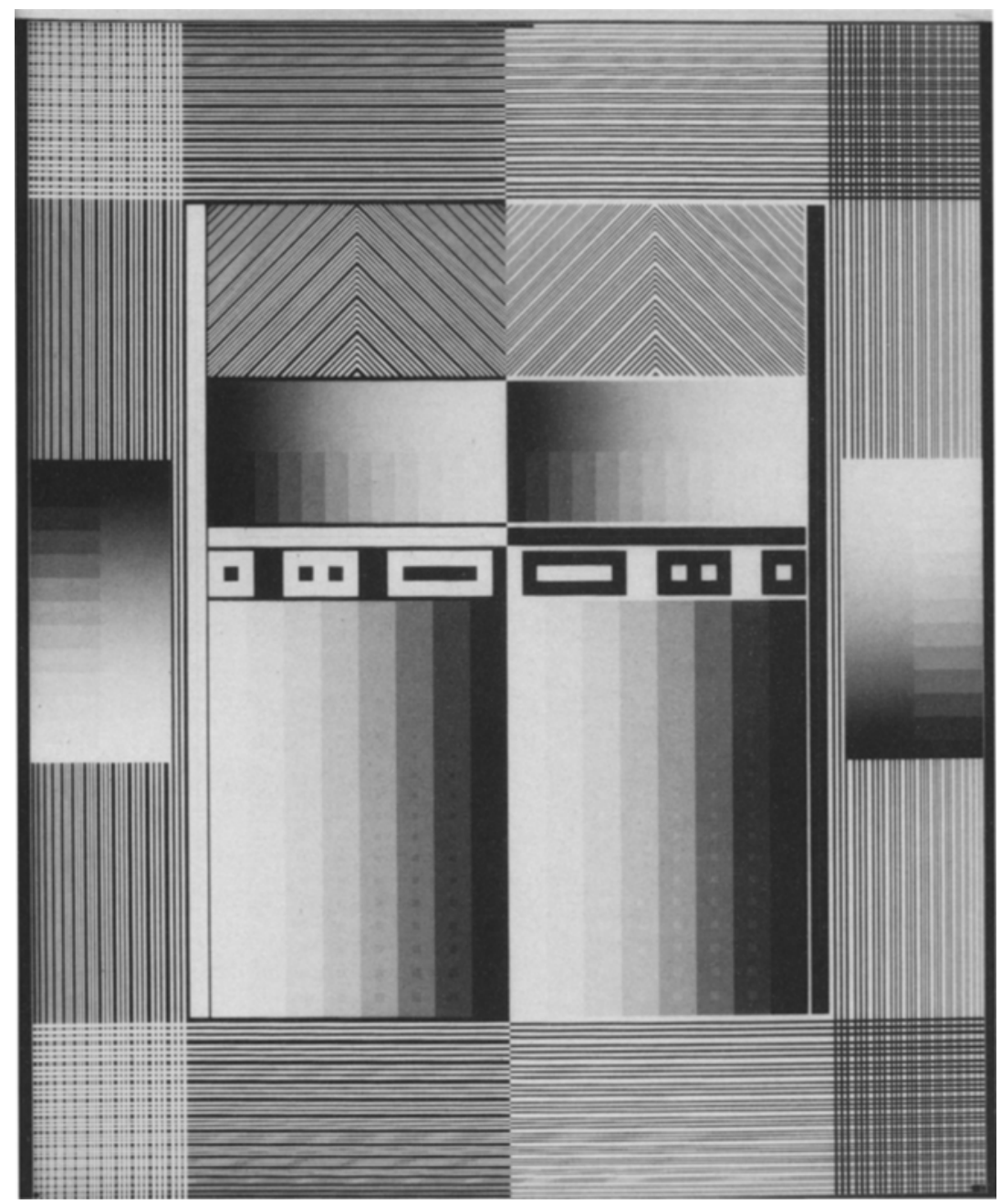

Fig 1. Quality control phantom. The design quantitates several basic parameters of image quality. Specific artifacts common to laser scanner digitizers are checked. 
Fig 2. Digitized image of the film phantom using the AT\&TPhilips CommView system. Digital pixel data was transferred to a personal computer for quantitative analysis.

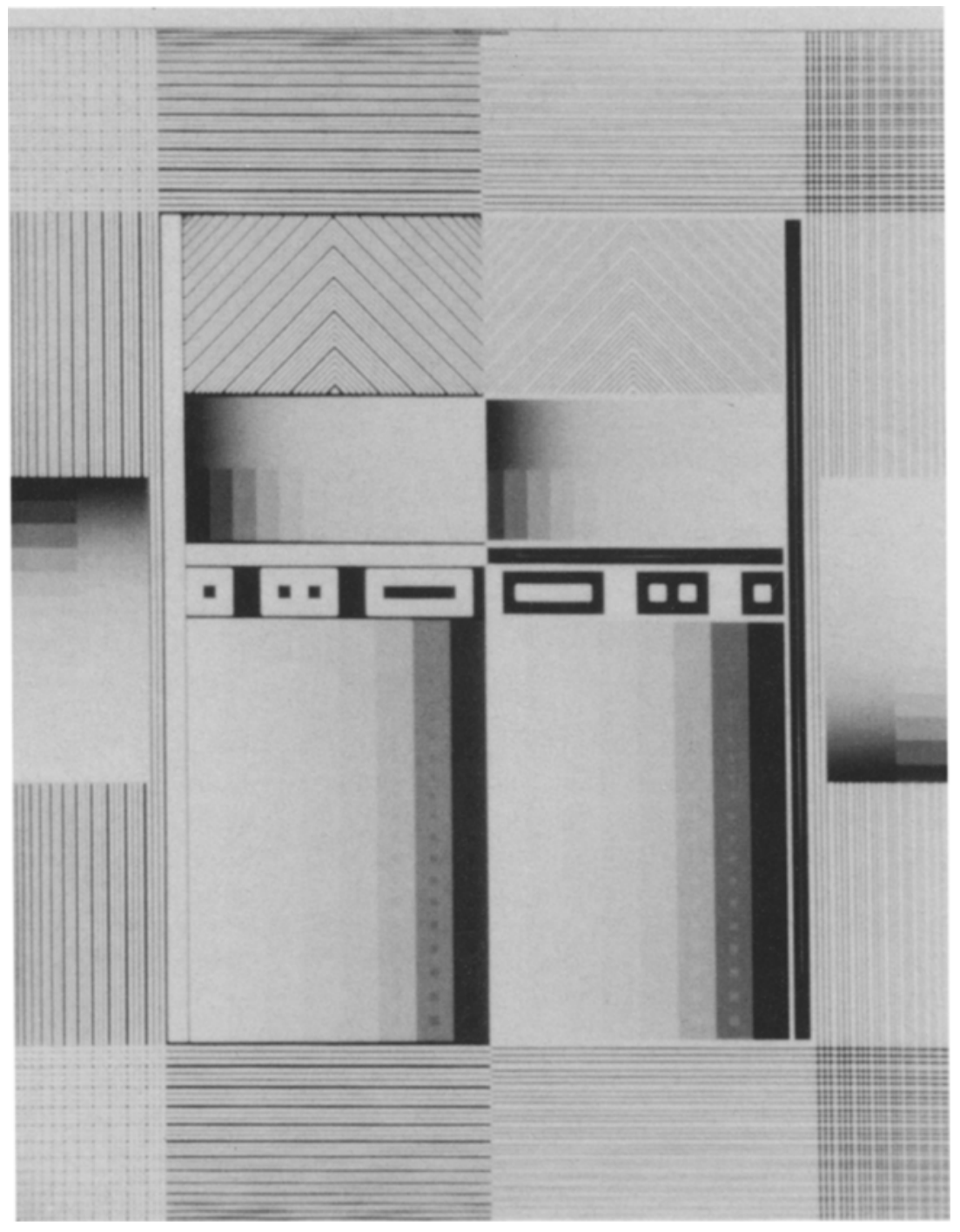

different density. On the left side of the image there are eight background strips ranging in density from 0.1 to 2.3 optical density units. Each strip contains 20 squares of diminishing size, which exceed the background density by 0.03 to 0.34 optical density units. (Larger differences in optical density are present in the darker strips.) The right side of the image holds the mirror image complement of these patterns so that the squares are of slightly less density than the surrounding background. The background strips range in density from 0.13 to 2.67 optical density units. The densities of the squares within these strips are 0.03 to 0.23 optical density units less than the background. Lowcontrast discrimination in various parts of the gray scale is quantified by simply noting the smallest square that can be seen at each background level when the phantom is digitized and displayed on a PACS system.

Gray scale performance is tested with both continuous and step wedge patterns located on either side of the phantom and across the midline (four sets of patterns in all). Continuous gray scale ramp and step wedge patterns are paired. The presence of multiple patterns permits the detection of differences in the digitized gray scale values in different portions of the image. Gray scale is also tested by the background strips in the low-contrast patterns. Qualitative analysis of the gray scale is accomplished by visually comparing the image display after 
digitization with the original gray scale on the film phantom. A quantitative assessment of linearity of the gray scale in the digitized image requires access to the digital data within the PACS system. The digitized pixel values may be plotted as a function of the optical densities within the step tablets on the original film (Fig $3)$.

Geometric distortion is tested in many parts of the phantom. There are straight lines present throughout the phantom in both horizontal and vertical orientations. In the center of the image there are several perfect squares, as well as rectangles with defined aspect ratios of 5:3 and $7: 3$. The diminishing squares in the low-contrast pattern should also be perfectly square in the digitized image.

The level of noise present in the digitized images of a PACS system can be approximated with the phantom if the digital image data of the PACS system is accessible. The noise is estimated as the standard deviation of digital pixel values within each of the various steps of the gray step tablets. Noise should be measured at multiple gray levels because it will generally increase with increasing density on the film.

Several features of the phantom were included to test for particular problems with laser scanner digitizers. (1) The ability to resolve long fine lines is tested in both the horizontal and vertical orientations, with black lines on a white background and white lines on a black background. (Fine line discrimination may be important for some radiological tasks such as detection of pneumothorax.) (2) Light saturation is tested by white squares and rectangles embedded in larger black squares and rectangles. Digitized images may exhibit streaking from bright areas that are immediately juxtaposed to darker areas. If this streaking is substantial it may obscure sharp light/dark interfaces. (3) Light leak is best detected along the $1 / 2$-inch black border on the sides of the phantom. If severe, light leak can diminish the diagnostic quality at the edges of digitized images.

The current phantom represents an improvement over our earlier phantom. ${ }^{12}$ The test patterns in the current phantom are more robust than those in our earlier design, and the laser printer produces a sharper image than our previous technique. High-contrast resolution patterns are located all around the periphery of the image in the present design, rather than just in the center. The line pairs are longer, extending the full length of each side of the film, thereby testing resolution in a larger portion of

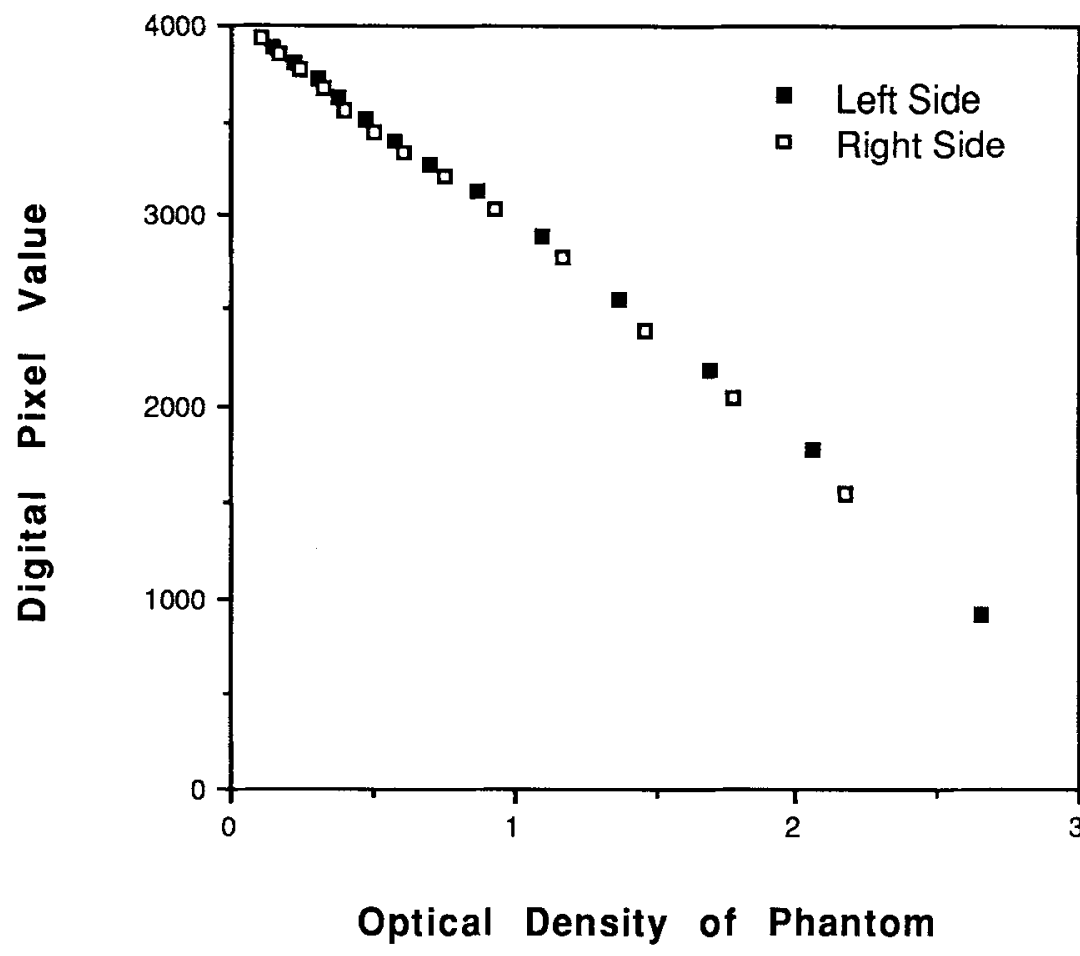

Fig 3. Digital pixel value as a function of optical density on the film phantom. Note the linear relationship that exists over the range of 0.1 to 2.7 optical density units. 


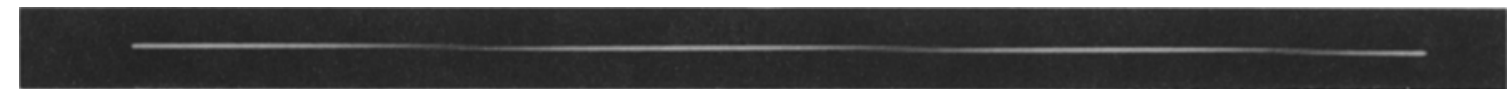

Fig 4. Fine line pattern following digitization with the CommView system. Note the loss of continuity of the line at regular intervals. This is an artifact introduced by laser scanner digitizers.

the image. The low contrast patterns have a greater number of different sizes of squares placed within the background, and a greater number of background gray levels. A continuous gray scale ramp has been added to the step tablet pattern present in the previous work. Several additional patterns have been incorporated to detect specific artifacts of laser scanner digitization as described above. By our own (subjective) visual assessment of the two film phantoms, the current design is sharper and has less mottle. A final significant advantage of using a laser printer rather than exposing the film directly is the fact that once the digital data file is created, many identical copies of the new phantom are quickly reproduced.

\section{TESTING A PACS SYSTEM}

The QC phantom we describe was used to test the CommView PACS system. The film phantom was digitized with the FD2000 laser scanner (DuPont, Wilmington, DE). It was transmitted from a remote location over a T-1 line and displayed on the AT\&T enhanced graphics display stations (Fig 2). The digitized image was also transmitted to PC-based workstations for further quantitative analysis.

Visual analysis showed consistent line pair discrimination along the entire length of the line pairs at $1.5 \mathrm{lp} / \mathrm{mm}$ in the horizontal, vertical, and diagonal directions. Low-contrast discrimination was present with all backgrounds at box sizes of $.16 \mathrm{~mm}$ or $.24 \mathrm{~mm}$. The gray scale was accurately reproduced in all parts of the image as judged by visual inspection. Analysis of the digitized image data (Fig 3) showed a linear relationship between optical density and digital pixel value over the range of 0.1 to 2.7 optical density units. No quantitative differences were found in the gray scale values digitized in

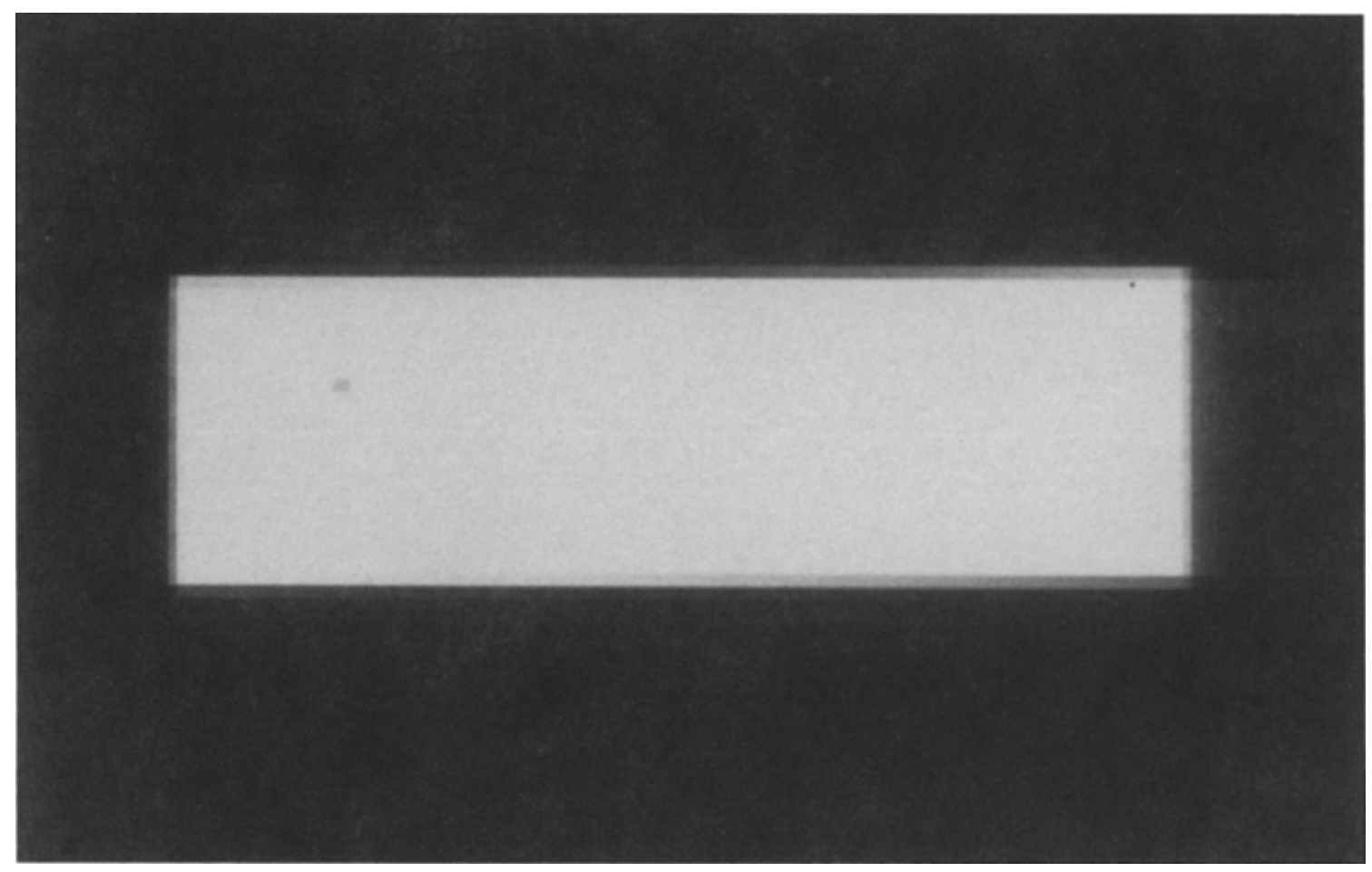

Fig 5. Bright rectangle surrounded by a dark border. The digitized image reveals streaking of light into the dark border on one side only. This results from saturation of the detectors as the scanner moves along the raster line. 
different parts of the image. There was no measurable geometric distortion. Noise, as quantified by the standard deviations within each step of the various step tablets, tended to be slightly greater in the darker portions of the gray scale. The standard deviation values ranged from 8 to 16 digital pixel units. Because each pixel contains 12 bits of data, a standard deviation of 3 to 4 bits suggests that there are 8 or 9 bits of noiseless data per pixel.

Three significant artifacts were discovered in the digitized image. (1) A regular pattern of pixel drop out was noted in the single pixel thickness lines (Fig 4). (2) Slight streaking was observed at the boundaries of light rectangles surrounded by black borders (Fig 5). The streaking was quantified by a difference of approximately 600 to 700 digital pixel units (on a scale of 4,096$)$ in different areas of the black border. (3) Light leak was noted along the left side of the digitized image (Fig 6). There was no significant light leak along the remaining edges.
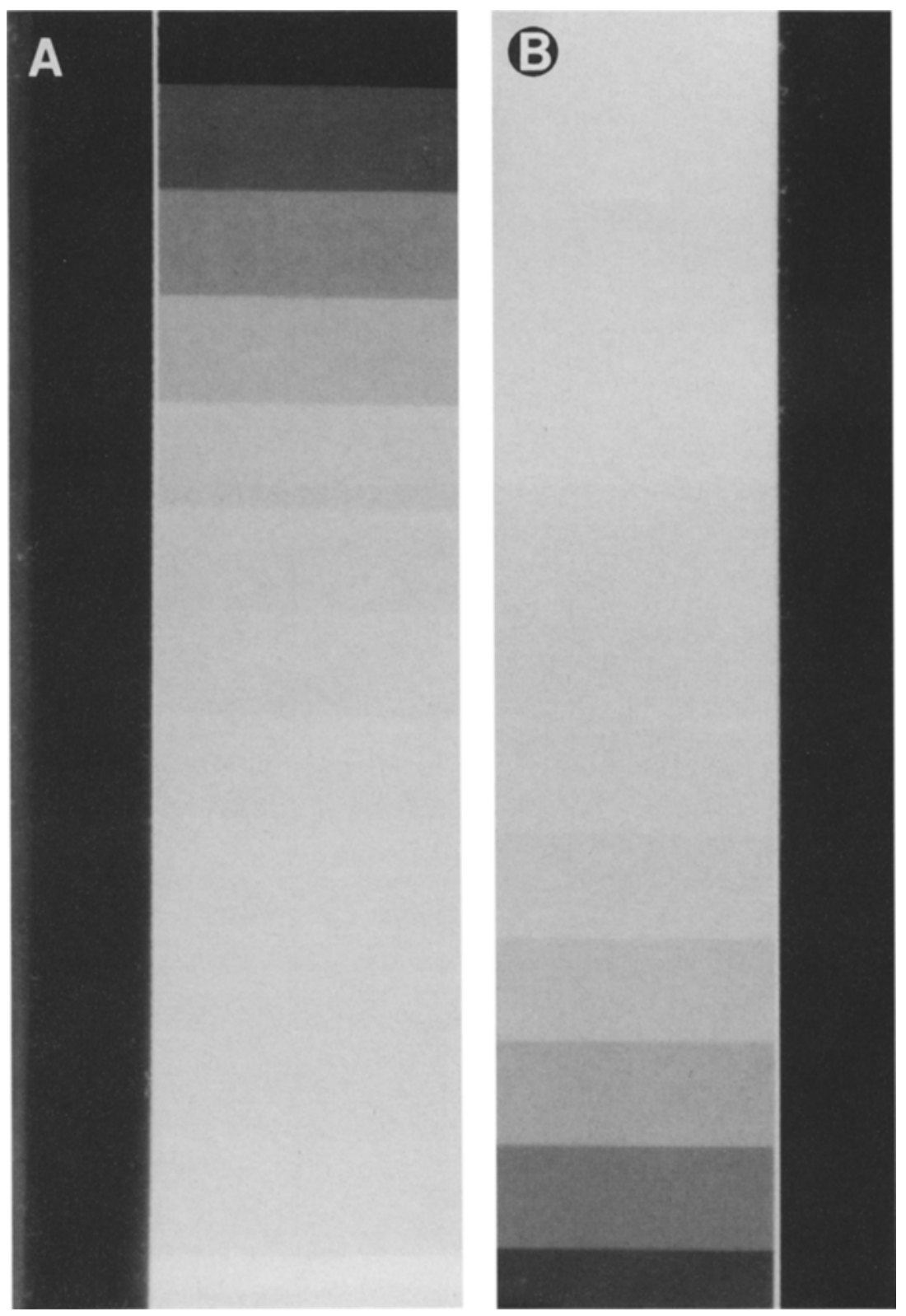

Fig 6. Left and right edges of the digitized image. Note that the light leak in this system is a much more significant problem on the left side of the digitized image. 


\section{DISCUSSION}

A regular QC protocol should be implemented for all clinical digital radiography systems to ensure appropriate diagnostic image quality. This protocol should include both phantom and clinical images. We have described a phantom image that is particularly appropriate for systems that digitize plain films. The phantom provides an objective, quantitative test of many of the important factors affecting image quality. It may also detect specific artifacts of plain film digitization.

This phantom is currently used as part of a daily QC protocol for the PACS system at our institution. By comparing the daily results, any change in system resolution or contrast definition is quickly detected. It also provides a simple and objective means of comparison with different PACS systems, and may thereby be of assistance in the initial choice of a PACS system for purchase.

Phantom images should be an integral part of any QC protocol for teleradiology and PACS.
However, it should be emphasized that synthetic test patterns such as our phantom quantify the system's ability to discriminate line pairs, low-contrast objects, and shades of gray, not its ability to provide accurate clinical diagnoses. These tests provide important objective data about the system, but they do not provide the answer to the ultimate question, "Is this system adequate for clinical diagnosis?" This question can be answered only by controlled clinical trials of the system in question. However, phantom test patterns remain most useful in the identification of specific problems with digital image quality.

\section{ACKNOWLEDGMENT}

We thank Michael A. Yancis of the AT\&T Bell Laboratories Medical Ventures group for his assistance in printing the quality control phantom and in providing the digital link between the AT\&T-Philips CommView system and our PC-based workstations, and George Knox for his assistance in digitizing and transmitting the phantom images used for this study.

\section{REFERENCES}

1. Takeuchi H, Taira RK, Huang HK, et al: Preliminary experience with a laser scanner and printer system for radiological image. Society of Photo-optical Instrumentation Engineers Proc PACS III 536:65-71, 1985

2. Lo SC, Taira RK, Mankovich NJ, et al: Performance characteristics of a laser scanner and laser printer system for radiological imaging. Comput Radiol 10:227-237, 1986

3. Vranck J, Strul B: Performance evaluation of a laser digitizer. Society of Photo-optical Instrumentation Engineers Proc Med Imaging 767:524-528, 1987

4. Lo SB, Gakill JW, Mun SK, et al: Contrast information of digital imaging in laser film digitizer and display monitor. J Digital Imaging 3:119-123, 1990

5. Halpern EJ, Esser PD, Nickoloff E, et al: Artifacts introduced by laser scanner digitization of radiographs, in Arenson RL, Fredenberg RM (eds): SCAR 90, Computer Applications to Assist Radiology (Proceedings of the 10th Conference on Computer Applications in Radiology). Anaheim, CA, Symposia Foundation, 1990, pp 276-284
6. Gray JE, Liks KG, Haddick DH, et al: Test pattern for video displays and hard-copy cameras. Radiology 154:519527, 1985

7. Roehrig H, Blume H, Ji TL, et al: Performance tests and quality control of cathode ray tube displays. J Digital Imaging 3:134-145, 1990

8. Dainty J, Shaw R: Image Science. San Diego, CA, Academic, 1974

9. Haus AG (ed): The Physics of Medical Imaging: Recording System Measurements and Techniques. New York, NY, American Association of Physicists in Medicine, 1979

10. Brody WR: Digital Radiography. New York, NY, Raven, 1984

11. Barrett $\mathrm{HH}$, Swindell W: Radiographic Imaging: The Theory of Formation Detection and Processing. San Diego, CA, Academic, 1981

12. Halpern EJ, Esser PD, Nickoloff EL, et al: A quality control phantom for digitization of radiographs. J Digital Imaging 3:42-48, 1990 